\title{
Pantothenic Acid
}

National Cancer Institute

\section{Source}

National Cancer Institute. Pantothenic Acid. NCI Thesaurus. Code C47783.

A water-soluble vitamin ubiquitously found in plants and animal tissues with antioxidant property. Vitamin B5 is a component of coenzyme A ( COA) and a part of the vitamin B2 complex. Vitamin B5 is a growth factor and is essential for various metabolic functions, including the metabolism of carbohydrates, proteins, and fatty acids. This vitamin is also involved in the synthesis of cholesterol, lipids, neurotransmitters, steroid hormones, and hemoglobin. 